\title{
Helicobacter pylori Gastritis: Susceptible to Further Testing?
}

\author{
Beverly B. Rogers ${ }^{1}$ Benjamin D. Gold ${ }^{2}$
}

Published online: 27 May 2016

(C) Springer Science+Business Media New York 2016

\section{Background and Significance}

Antibiotic resistance has reached crisis proportions in the USA. A report from the Antibiotic Resistance Working Group of The President's Council of Advisors on Science and Technology [1] states that the "Centers for Disease Control and Prevention (CDC) and the World Health Organization (WHO) each recently identified antibiotic resistance as one of the greatest threats to human health worldwide. CDC estimates that each year more than two million people in the United States are sickened with infections by more than 17 types of antibiotic-resistant microbes." Interestingly, of the 18 organisms specifically cited in the report, none was $H$. pylori.

The prevalence of $H$. pylori in the general US population is unknown; in 1999-2000, $25.4 \%$ of the US population was seropositive [2]. Other studies described a prevalence rate of 30-40\% in US adults and a 60-70\% infection rates in immigrant populations and those of lower socioeconomic status and living under crowded circumstances [3, 4]. Resistance to clarithromycin, one of the components of recommended first-line therapy, has been increasing, with 10-15\% resistance in 2004 [5] and $\sim 30 \%$ resistance reported in the article by Park et al. [6] in this issue of Digestive Diseases and Sciences. Given that

Beverly B. Rogers

beverly.rogers@choa.org

1 Department of Pathology, Children's Healthcare of Atlanta and Emory University School of Medicine, Atlanta, GA, USA

2 Children's Center for Digestive Healthcare, LLC, Emory University School of Medicine, 993-D Johnson Ferry Road, NE, Suite 440, Atlanta, GA 30342, USA
$320,000,000$ people live in the USA, the number of persons infected with clarithromycin-resistant $H$. pylori is estimated in the hundreds of thousands, similar or greater than the estimated annual infections by carbapenem-resistant or $\beta$-lactamase-producing Enterobacteriaceae $(35,300)$, drugresistant Campylobacter $(310,000)$, or vancomycin-resistant Enterococcus $(20,000)$ [1].

As with the prevalence rates of $H$. pylori infection, resistance rates, a major factor in determining the success of eradication therapy, also vary worldwide depending on geographic regions. In many countries, the primary antimicrobial resistance to clarithromycin has increased to levels above the recommended threshold for use as a firstline agent for $H$. pylori infection. In a recent nationwide population-based Korean study, the antibiotic resistance rates of $H$. pylori were distinct in different regions. The authors strongly recommended that local data should be provided as a guideline for treating $H$. pylori infection. In a Vietnamese study, clarithromycin resistance, which exceeded $50 \%$ in young children, was a significant cause of treatment failure. The authors suggested that clarithromycin should not be used as part of $H$. pylori eradication therapy in children if their antibiotic susceptibility profile is unknown [7-10]. The conclusions of the authors from these aforementioned studies reflect a distinct change in the approach to management of $H$. pylori infection, which is targeted therapy based on resistance profiles, rather than the disease outcomes. Furthermore, and closer to home, a recent review from Latin America described high rates of resistance of $H$. pylori to first-line antibiotics; the authors recommended susceptibility testing prior to the use of clarithromycin [11]. Therefore, treatment failure, primarily due to increasing resistance rates, raises the cost of $H$. pylori infection management and the burden of this infection and associated disease to the patient and their 
family. The inability to initially eradicate $H$. pylori infection will increase healthcare utilization, increasing the number of unnecessary procedures and therapies and their associated risks. Moreover, eradication failure increases the risk of antibiotic resistance of the infecting $H$. pylori strains, thereby reducing the likelihood of successful eradication.

Helicobacter pylori is not only an infectious organism, but also an oncogenic agent. The WHO has classified $H$. pylori as a class 1 carcinogen based on data that support the contention that this organism meets all of Koch's postulates as a human gastric carcinogen. With a near threefold increase in gastric carcinoma among patients who are seropositive for $H$. pylori, eradication of the infection decreases the incidence of cancer development [12]. In "atrisk" H. pylori-infected populations, without an effective vaccine, there is a need to treat with appropriate, highly effective and targeted therapy to eradicate the organism in infected individuals.

\section{How the Article Addresses the Controversies}

One clear message highlighted by Park et al. [6] is that the current rate of clarithromycin resistance for $H$. pylori in the USA exceeds the rate at which empiric therapy is recommended, yet empiric therapy is the standard treatment for $H$. pylori gastritis. The authors report a $>20 \%$ rate of clarithromycin-resistant $H$. pylori gastric infection, identified through genetic sequencing of $H$. pylori DNA extracted from paraffin-embedded tissue sections submitted from four locations across the USA. The prevalence of clarithromycin resistance varied from 23.1 to $45.8 \%$; the only factor impacting the resistance rate was a history of prior treatment failure. There have been numerous reports of similar or higher resistance rates to clarithromycin throughout the world and in specific locations in the USA, but this is the first article to assess prevalence in multiple locations, therefore identifying clarithromycin-resistant $H$. pylori as an important public health problem. Thus, the conceptual approach to management of $H$. pylori infection-associated disease by gastroenterologists, which has been to resolve the disease outcome, must change to one that addresses the infection as an infectious disease.

To expand on the discussion in the article, the 2007 management guideline for $H$. pylori infections from the American College of Gastroenterology notes that the efficacy of first-line treatment with a proton pump inhibitor (PPI), clarithromycin, and amoxicillin has decreased to $70-85 \%$, in part due to clarithromycin resistance [13]. The 2007 article states that poor compliance and antibiotic resistance are the most important predictors of treatment failure, citing articles published in 2002 [14] and 2004 [5] describing $>20 \%$ resistance for metronidazole, $<2 \%$ resistance for amoxicillin, and 10-13\% resistance for clarithromycin.

Park et al. advise that "antibiotic resistance testing, in particular for clarithromycin resistance, of individuals diagnosed with $H$. pylori infection should be performed to help tailor antibiotic therapy to a regimen with a high likelihood of success." This recommendation then reflects a change in management strategy-from one designed to resolve disease - to one that is designed more appropriately to treat the infection. Antibiotic resistance of $H$. pylori strains, though, is not limited to clarithromycin. The following rates of $H$. pylori resistance were reported in a 2015 report from Shanghai, China: clarithromycin $40.0 \%$, amoxicillin $4.4 \%$, metronidazole $53.3 \%$, tetracycline $0 \%$, and levofloxacin $55.6 \%$ [15]. While testing for clarithromycin resistance alone will provide important information, to treat $H$. pylori gastritis as an infectious disease will require available testing for not only clarithromycin, but also for other commonly used antibiotics. Studies are beginning to appear that demonstrate more acceptable eradication rates when therapy is tailored according to the antimicrobial susceptibility profile, rather than the traditional empiric therapy that has been used for the past 25 years [16]. Despite the knowledge of resistance, there was, counterintuitively, no strong recommendation made by the American College of Gastroenterology for antibiotic resistance testing to be performed prior to prescribing antibiotics [13]. The lack of readily available antibiotic susceptibility testing methods makes treating $H$. pylori like an infectious disease difficult. Culture of $H$. pylori requires a strict microaerophilic environment with $5 \% \mathrm{CO}_{2}$ at $35{ }^{\circ} \mathrm{C}$ for 3-7 days, complicating identification and antibiotic resistance testing [17]. With many clinical laboratories unable to grow the organism and perform the current FDA-recommended method for antimicrobial resistance testing, namely agar dilution, targeted eradication strategies for $H$. pylori-infected persons have not been undertaken as part of a routine treatment plan.

Several molecular assays have been developed to identify the bacterial genetic alterations conferring resistance for the antibiotics used to treat $H$. pylori, providing similar susceptibility profiles when compared to culture-based methodology [18-21]. While some of the molecular assays are in clinical use, such as the assay used by Park et al., the methods are not used in most clinical laboratories because of the complexity of the assays. Furthermore, to test all of the antibiotics used to treat $H$. pylori for resistance would require assays for multiple gene targets and, therefore, additional complexity. 


\section{Summary and Future Directions}

It is time to treat $H$. pylori infections with therapy that is appropriate, using precision medicine to match antibiotic therapy with the resistance pattern. To do this will require readily available diagnostic testing for the confirmation of infection, and the ability to perform timely antibiotic resistance testing. Empiric treatment, no longer appropriate in many areas due to the high level of antibiotic resistance, must be supplanted by antibiotic resistance testing or by alternative first-line therapy. Soon-to-be published guidelines for the management of $H$. pylori infection in children will specifically recommend pre-treatment susceptibility profiles, a knowledge (if available) of prevalence trends for $H$. pylori strains from within the region, and targeted, highly effective eradication therapy (i.e., eradication rates $>90 \%$ ). Furthermore, due to the unacceptably low $H$. pylori eradication rates in children and adults reported in the literature from different countries, as well as the increasing rates of antibiotic resistance among all subjects of all ages, treatment for $H$. pylori in children as well as adults should be provided when it is clinically indicated, and with the most effective therapy available-one which is based on the susceptibility profiles of the infecting strain. Until the time when the technology evolves to the point that antibiotic resistance testing for this organism is routine for all clinical laboratories, local prevalence should be determined and, if this exceeds $20 \%$, susceptibility testing for clarithromycin, at the least, should be requested from those laboratories that are able to perform the assay. Laboratories should work diligently to develop methodologies (a "gold standard") that employ molecular tools to enable rapid, efficient, highly robust testing of infecting $H$. pylori strains to then offer effective, targeted eradication therapy.

Future studies should focus on obtaining antibiotic resistance rates across regions to help direct optimal therapy, and monitor trends in resistance. Furthermore, highquality studies evaluating novel approaches and therapies including concomitant therapy and the contribution of supplemental probiotics are needed. Importantly, studies of prevention of infection with optimization of vaccine strategies should be performed such that the use of antibiotics for $H$. pylori infection can be curtailed, particularly in highly prevalent areas that also have substantial disease burdens from this chronic human infection.

\section{References}

1. Report to the President on Combatting Antibiotic Resistance. President's Council of Advisors on Science and Technology.
September 2014. https://www.whitehouse.gov/sites/default/files/ microsites/ostp/PCAST/pcast_amr_jan2015.pdf.

2. Krueger WS, Hilborn ED, Converse RR, Wade TJ. Environmental risk factors associated with Helicobacter pylori seroprevalence in the United States: a cross-sectional analysis of NHANES data. Epidemiol Infect. 2015;143:2520-2531.

3. Rosenberg JJ. Helicobacter pylori. Pediatr Rev. 2010;31:85-86. discussion 86.

4. Roberts SE et al. Review article: the prevalence of Helicobacter pylori and the incidence of gastric cancer across Europe. Aliment Pharmacol Ther. 2016;43:334. http://www.jwatch.org/na40142/ 2016/01/19/prevalence-helicobacter-pylori-and-incidence-gastric\# sthash.LZoAhX4F.dpuf.

5. Duck WM, Sobel J, Pruckler JM, et al. Antimicrobial resistance incidence and risk factors among Helicobacter pylori-infected person, United States. Emerg Infect Dis. 2004;10:1088-1094.

6. Park JY, Dunbar KB, Mitui M, et al. Helicobacter pylori clarithromycin resistance and treatment failure are common in the United States. Dig Dis Sci. (Epub ahead of print). doi:10.1007/ s10620-016-4091-8.

7. Megraud F, Coenen S, Versporten A, et al. Helicobacter pylori resistance to antibiotics in Europe and its relationship to antibiotic consumption. Gut. 2013;62:34-42.

8. Miendje Deyi VY, Bontems P, Vanderpas J, et al. Multicenter survey of routine determinations of resistance of Helicobacter pylori to antimicrobials over the last 20 years (1990 to 2009) in Belgium. J Clin Microbiol. 2011;49:2200-2209.

9. Seo JH, Jun JS, Yeom JS, et al. Changing pattern of antibiotic resistance of Helicobacter pylori in children during 20 years in Jinju, South Korea. Pediatr Int. 2013;55:332-336.

10. Nguyen TV, Bengtsson C, Yin L, et al. Eradication of Helicobacter pylori in children in Vietnam in relation to antibiotic resistance. Helicobacter. 2012;17:319-325.

11. Camargo M, et al. The problem of Helicobactor pylori resistance to antibiotics: a systematic review in Latin America. Am J Gastroenterol. 2014;109:485-495.

12. Sitas F. Twenty-five years since the first prospective study by Forman et al. (1991) on Helicobacter pylori and stomach cancer risk. Cancer Epidemiol. 2016;41:159-164.

13. Chey WD, Wong BC, Practice Parameters Committee of the American College of Gastroenterology. American College of Gastroenterology guideline on the management of Helicobacter pylori infection. Am J Gastroenterol. 2007;102:1808-1825.

14. Meyer JM, Silliman NP, Wang W, et al. Risk factors for Helicobacter pylori resistance in the United States: the surveillance of H. pylori antimicrobial resistance partnership (SHARP) study, 1993-1999. Ann Intern Med. 2002;136:13-24.

15. Dong F, Ji D, Huang R, et al. Multiple genetic analysis systembased antibiotic susceptibility testing in Helicobacter pylori and high eradication rate with phenotypic resistance-guided quadruple therapy. Medicine. 2015;94:1-7.

16. Li BZ, Threapleton DE, Wang JY, et al. Comparative effectiveness and tolerance of treatments for Helicobacter pylori: systematic review and network meta-analysis. BMJ. 2015;351: h4052.

17. Jerris RC. Helicobacter pylori. In: Leber AL (ed.). Clinical microbiology procedure handbook. Washington, DC: ASM Press; 2004.

18. Stubbs SLJ, Brazier JS, Talbot PR, Duerden BI. PCR restriction fragment length polymorphism analysis for identification of Bacteroides spp. and characterization of nitroimidazole resistance genes. J Clin Microbiol. 2000;38:3209-3213.

19. Trespalacios AA, Rimbara E, Otero W, Reddy R, Graham DY. Improved allele-specific PCR assays for detection of clarithromycin and fluoroquinolone resistant of Helicobacter pylori 
in gastric biopsies: identification of N87I mutation in GyrA. Diagn Microbiol Infect Dis. 2015;81:251-255.

20. Lawson AJ, Elviss NC, Owen RJ. Real-time PCR detection and frequency of $16 \mathrm{~S}$ rDNA mutations associated with resistance and reduced susceptibility to tetracycline in Helicobacter pylori from England and Wales. J Antimicrob Chemother. 2005;56:282-286.
21. Versalovic J, Osato MS, Spakovsky K, et al. Point mutations in the 23S rRNA gene of Helicobacter pylori associated with different levels of clarithromycin resistance. J Antimicrob Chemother. 1997;40:283-286. 